\title{
Status and Vocal Accommodation in Small Groups
}

Joseph Dippong

University of North Carolina at Charlotte

Abstract: Sociological research on vocal dynamics demonstrates that as actors engage in conversation, their vocal frequencies tend to converge over time. Previous scholars have theorized that patterns of vocal accommodation serve as a mechanism through which speakers non-consciously communicate perceptions of each other's relative status. In this article I discuss existing evidence linking vocal accommodation to status perceptions. Next, I report results from a laboratory experiment in which I test the proposed causal link between status and vocal accommodation by randomly assigning members of dyadic task groups to occupy either a higher or lower status position and assessing patterns of vocal accommodation. Results support the theorized causal relationship between group status structure and vocal accommodation. I argue that, as an unobtrusive and non-conscious indicator of status, vocal accommodation is a valuable, yet underused tool for assessing group status structures in a wide variety of situations and regarding many substantive sociological questions.

Keywords: status; prestige; dominance; vocal accommodation; measurement

Citation: Dippong, Joseph. 2020. "Status and Vocal Accommodation in Small Groups." Sociological Science 7: 291-313.

Received: May 6, 2020

Accepted: June 8, 2020

Published: August 3, 2020

Editor(s): Jesper Sørensen, Mario Small

DOI: $10.15195 /$ v7.a12

Copyright: (C) 2020 The Author(s). This open-access article has been published under a Creative Commons Attribution License, which allows unrestricted use, distribution and reproduction, in any form, as long as the original author and source have been credited. (0)(1)
$\mathrm{R}$ ECENTLY, scholars across the social sciences have begun to take an increased interest in the role of vocal dynamics in social interaction. A growing body of research points to the fact that as people interact, their voices convey much more information than they intend or even realize. Regarding overt vocal characteristics, Pitts and Gallois (2019) discuss the various ways that dialect, social class differences in speech, and age and sex markers in the voice influence the processes through which people categorize interaction partners. Regarding more subtle vocal characteristics, evidence indicates that people non-consciously attend to a variety of acoustic markers in the voice-such as pitch and fundamental frequency-that allow them to make rapid inferences about personality and trustworthiness; such inferences arise even after a single spoken word (Baus et al. 2019). And in terms of more structural aspects of speech, research on linguistic alignment shows that people line up their speech patterns with those of their interaction partners as a means to signal social closeness (Ruch, Zürcher, and Burkart 2018).

Whereas psychologists, linguists, and communications scholars have focused largely on how various vocal properties shape the perceptions and inferences people form about others, sociologists have focused primarily on examining what vocal dynamics reveal about micro-level structures of status and power in small groups (e.g., Gregory 1994; Kalkhoff, Thye, and Gregory 2017). Along these lines, one promising line of research suggests that between-actor convergence in vocal frequencies-a process called vocal accommodation-serves as a channel through which group members non-consciously communicate perceptions of each other's relative status. Given the proposed relationship between vocal dynamics and small group hierarchies, recent theoretical and empirical developments point to the intriguing possibility that researchers can unobtrusively assess and model 
status structures using established measures of vocal accommodation (Dippong and Kalkhoff 2018). As a methodological tool, vocal analysis has broad potential for examining processes and outcomes of interest to sociologists who study social psychology, organizations, inequality, communication, and biosocial processes.

Although the existing research connecting vocal accommodation to group status structures is noteworthy, at least two substantial issues require further examination. First, based on methodological considerations, the vast majority of sociological research in this area has relied on quasi-experimental and survey-based approaches that infer group structures backward from observed patterns of vocal accommodation, most often using post hoc measures collected from panels of external observers outside the group (e.g., Gregory and Webster 1996; Gallagher et al. 2005). The reliance on observers' ratings has created a research model in which more often than not, researchers begin with observed accommodation differences in groups where the status structure is unknown and then predict the status or dominance perceptions of actors who are exterior to the group. Second, the practice of inferring structure from posterior rankings has made it difficult to separate the effects of status from the effects of dominance displays, which represent two distinct foundations of group hierarchy (Cheng et al. 2013; Cheng and Tracy 2014). Even studies that have attempted to disentangle the unique effects of status and dominance on vocal accommodation (e.g., Kalkhoff et al. 2017) have done so essentially blind to the underlying basis of the group hierarchy.

Although prior work has produced impressive results, a strong test of the causal link between status and vocal accommodation requires measuring patterns of vocal accommodation in interactions that meet the following three criteria: (1) the group is organized according to a hierarchy of status rather than dominance; (2) the status structure of the group is known, allowing us to predict behavior from structure and not vice versa; and (3) accommodation differences can be mapped onto the status perceptions of group members themselves, rather than the perceptions of external observers (Dippong and Kalkhoff 2018). The present study examines vocal accommodation under such conditions.

In what follows, I present the results of a laboratory experiment examining the relationship between vocal accommodation and small group status structures. The primary purpose of this study is to demonstrate a causal relationship between actors' relative status in a group and the patterns of vocal accommodation that emerge between speakers. I also seek to establish vocal accommodation as a viable unobtrusive measure of group status structure that researchers can use either alongside existing measures of status or as an alternative to traditional approaches. I begin with a brief overview of the theoretical and empirical background linking vocal accommodation and status. Next, I describe a laboratory experiment that tests three central aspects of vocal accommodation: (1) the causal impact of status on vocal accommodation in a setting in which status differences are established prior to group interaction; (2) the relationship between vocal accommodation and group members' subjective perceptions of the group status structure; and (3) the extent to which measures of vocal accommodation are sensitive to magnitudes of status differentiation, comparing groups with varying degrees of status differences between interactants. After presenting my results, I conclude with a discussion of 
the implications of the findings, highlighting the potential value of vocal accommodation as an unobtrusive measure of status, and outline some future directions and unanswered questions.

\section{Theoretical and Empirical Background}

Current sociological models of vocal accommodation draw heavily on theory and research not just from sociology, but also from research on communication and linguistics. Communication scholars have long observed that as individuals interact, their behaviors tend to converge. For example, two people dining together in a restaurant will often seem instinctively to take a drink at the same time; likewise, when one person in a group crosses their arms or legs, others nearby tend to follow suit. This process of interactional synchrony (Condon and Sander 1974) can generate a behavioral rhythm that coordinates the actions of those involved and enhances solidarity between actors (Hall 1981; Kalkhoff, Dippong, and Gregory 2011; Kalkhoff et al. 2020). Observable aspects of interactional synchrony include alignment in posture and gestures (Condon and Ogsten 1967) and in speech patterns, speaking rate, and "tone" (Natale 1975). When actors converge in their speech and behavior, it promotes smooth interaction and minimizes the impact of social differences between people (Giles and Coupland 1991).

The tendency to "sync up" with interaction partners appears to reflect processes that are non-conscious (Condon and Sander 1974) and may be a fundamental component of social interaction (Condon 1986). As evidence that synchrony occurs outside of conscious awareness or control, Condon and Ogsten (1967) found that just as actors converge in their outward actions, they also demonstrate a form of inward synchronization in internal biological processes. Condon and Ogsten found evidence for inward synchrony in patterns of shared electrical activity in the brains of interactors (as measured by electroencephalogram), and more recent research has verified the existence of such interbrain synchronization (Kalkhoff et al. 2015). As such, synchrony occurs in actions of which individuals are consciously aware and in behaviors that are beyond conscious control.

Working within the context of interactional synchrony, Gregory (1983; Gregory and Hoyt 1982) identified an additional form of inward synchrony reflected in convergence between speakers' vocal frequencies. Gregory (1983) observed that over the course of a dyadic conversation, speakers' vocal frequencies converge to produce a "vocal fingerprint" that is unique to the interaction. Like other manifestations of synchrony, vocal convergence may constitute a fundamental component of human speech. Although researchers have focused primarily on documenting synchrony in the vocal frequencies of English language speakers, evidence from one study involving Arabic-speaking groups in Egypt revealed the same level of vocal synchrony as that typically observed in American English-speaking groups (Gregory, Webster, and Huang 1993). Thus, vocal synchrony is not merely a byproduct of a specific language but is likely a process that is agnostic to language and culture.

Vocal convergence occurs across a wide range of frequencies, although evidence suggests that synchrony is strongest in frequencies below $500 \mathrm{~Hz}$-a segment of 
the vocal spectrum known as the paraverbal frequency band (Gregory, Dagan, and Webster 1997; Gregory et al. 2001). The paraverbal frequencies reflect properties that people typically associate with vocal pitch, tone of voice, and emotion. That the strongest convergence occurs within the paraverbal band underscores the notion that synchrony reflects non-conscious processes; unlike frequencies within the verbal band, it is very difficult to consciously manipulate the paraverbal frequencies (Bachorowski 1999).

\section{Vocal Synchrony and Vocal Accommodation}

Building on scholarship on behavioral convergence and synchrony, research within the framework of accommodation theory examines how features of the interactional setting affect the convergence process (Giles and Coupland 1991; Giles and Smith 1979). ${ }^{1}$ Accommodation theory observes that as individuals adapt to each other and adopt each other's mannerisms and behaviors, synchrony often arises unequally, with one interaction partner "putting in more work" to adapt and converge (Gallois, Ogay, and Giles 2005). In essence, if interaction has a rhythm, accommodation theory suggests that there is often a "conductor" who establishes the tempo and rhythm that others follow. Furthermore, structural aspects of the interactional setting, such as status and power differences between actors, influence which group member becomes the behavioral conductor of the interaction. Lower status actors converge toward higher status actors much more than the other way around, and this difference in convergence effort is called accommodation. Although all group members benefit from the effects of synchrony-including greater solidarity (Kalkhoff et al. 2011), communication quality (Gregory et al. 1997), and cohesion (Gregory et al. 2009)—evidence suggests that these benefits arise largely through the accommodative efforts of lower status actors.

Recognizing that lower status actors put in greater effort to adapt than do their higher status counterparts, the central claims of accommodation theory map neatly onto research on vocal synchrony. ${ }^{2}$ That is, as group members converge in their paraverbal vocal frequencies, accommodation theory suggests that lower status actors will move toward higher status actors more so than the other way around. Across a number of studies, researchers have consistently found that convergence in vocal signals in dyadic groups occurs unequally, with one actor demonstrating greater paraverbal variability than the other (Gregory 1994; Gregory and Gallagher 2002; Kalkhoff and Gregory 2008). Additional studies have shown that observers who listen to such conversations attribute lower status to speakers who demonstrate greater paraverbal variability. Evidence suggests, then, that status differences produce differences in vocal accommodation.

In an early test of the vocal accommodation model, Gregory and Webster (1996) analyzed a series of interviews between talk show host Larry King and guests on his televised program, including politicians, athletes, and other celebrities. Results indicated that the interviewer adapted vocally to higher status partners while demonstrating greater vocal stability when interviewing relatively lower status guests. Gallagher and colleagues (2005) further developed the vocal status model, examining interactions between medical students and simulated patients, finding 
that asymmetry in vocal convergence emerged when the interaction centered on tasks related to the interviewers' area of expertise, suggesting that the process was driven by status and prestige differences between speakers. More recently, Moore, Dippong, and Rejtig (2019) provide evidence that in mixed-sex groups, female participants assigned to task leadership positions demonstrate less paraverbal variability than those assigned to non-leadership positions.

In an effort to differentiate the effects of prestige and dominance on vocal accommodation, Kalkhoff and colleagues (2017) analyzed vocal data from televised debates between talk show host Piers Morgan and three different guests. Kalkhoff et al. (2017) measured viewers' perceptions of each actor's dominance and prestige, finding that paraverbal variability significantly predicted observers' dominance perceptions of the speakers, but not their perceptions of prestige. In the absence of specific knowledge of the underlying status structure of the interview dyads, however, it is unclear whether the interactional hierarchies represent status structures or dominance structures. Nonetheless, Kalkhoff and colleagues (2017) show that accommodation differences reflect hierarchical differences between group members generally.

None of the studies discussed here conclusively demonstrates a causal relationship between vocal accommodation behaviors and relative position within a small group status hierarchy, although each provides a piece of the puzzle. Building on accommodation theory, which argues that lower status actors contribute greater effort toward behavioral convergence, coupled with findings related to interviewers' greater accommodation when interviewing higher status guests and evidence that patterns of vocal accommodation reflect status perceptions, I offer the following hypothesis:

$\mathrm{H}_{1}$ : Relative status position within a group is positively related to vocal accommodation behaviors.

Specifically, I aim to demonstrate the causal effect of status differences on vocal accommodation. I test my hypothesis using two different indicators of status position: participants' assigned positions within an induced status hierarchy created through experimental manipulation, and self-reported perceptions of status relative to their interaction partner.

Because I am also interested in demonstrating the viability of vocal accommodation as an unobtrusive measure of status, I also test whether measures of vocal accommodation are sensitive to magnitudes of status differentiation between actors. To date, little is known about important measurement properties of common indicators of vocal accommodation in sociological research. To this end, it is possible that vocal accommodation distinguishes group members in one of two different ways: (1) as a rough binary higher-versus-lower status indicator, producing similar measurements for large and small status differences or (2) as a more fine-grained indicator, with larger status differences between group members producing larger differences in accommodation measures. To examine whether the measure I employ is sensitive to varying magnitudes of status differentiation, I test one additional prediction: 
$\mathrm{H}_{2}$ : As status differences between speakers increase, differences between speakers' vocal accommodation scores will also increase.

Because prior work has yet to directly address the issue of measurement sensitivity to magnitudes of status differentiation, testing hypothesis 2 will provide additional insight and clarification regarding the causal relationship between status and vocal accommodation.

\section{A Note on Analyzing Vocal Data}

Over time, researchers who study vocal dynamics have developed and refined standard methods for analyzing data and assessing accommodation, and these analytical procedures are available in a variety of published sources. ${ }^{3}$ Assessing vocal accommodation involves first converting the audio signal of speakers' voices into quantitative values using fast Fourier transform (FFT) analysis. In short, FFT functions much like a prism that separates visible light into distinct color wavelengths (Gregory 1986). FFT splits the complex waveform of the human voice into hundreds of separate frequencies, summarizing the audio signal by plotting acoustic frequencies for a given utterance against the average amplitude at that frequency (Gregory and Kalkhoff 2007).

After converting speakers' acoustic signals via FFT, researchers can isolate the paraverbal frequencies of the voice and calculate a variety of measures. The earliest research on vocal dynamics employed simple bivariate correlations between speakers' FFT signals to model synchrony, with positive correlations signifying convergence (Gregory and Hoyt 1982; Gregory 1983). Subsequent research has utilized principle components analysis of FFT values to produce a measure of vocal stability known as acoustic analysis result (AAR). The logic behind the AAR measure is that in interactions where actors' vocal frequencies converge, speakers who accommodate their partners will demonstrate less vocal stability. If status differences are driving the accommodation process, we should expect lower status actors to produce lower AAR values.

When vocal accommodation has occurred, the data should reflect two properties: (1) a positive correlation between speakers' FFT values and (2) a difference in variability/stability between speakers' paraverbal frequencies across the duration of interaction, with higher AAR values denoting greater stability and lower values indicating greater paraverbal variability. Regarding AAR, Kalkhoff et al. (2017:1) state, "The FFT measure of vocal adaptation is useful insofar as it is relatively easily obtained, objectively measured, reliable, and taps into a quantitative property of conversational speech that seems to occur outside of people's conscious control." As such, vocal measures possess a number of desirable properties.

\section{Method}

To examine the relationship between status structure and patterns of vocal accommodation, I conducted a laboratory experiment in which I randomly assigned members of dyadic discussion groups to occupy either a higher or lower status position. 
Following the status manipulation, group members completed an open-interaction decision-making task, and I collected audio recordings of the discussions. I predict that under conditions of vocal convergence, lower status actors will demonstrate greater paraverbal variability. Furthermore, I anticipate that as status differences increase, between-actor differences in paraverbal variability will also increase. Unlike previous work that employs panels of external raters to infer the status structure of a group they observe, the present study models the status-accommodation link using only the manipulated structure of the group and the overt behavior of interacting actors.

\section{Participants}

A total of 92 student volunteers participated in the study, which took place at a large Southeastern university. Participants signed up for research sessions using an online appointment scheduling platform, with arrival times staggered to reduce the possibility of interaction between group members prior to the study. All participants completed the study in same-sex, two-actor groups. I randomly assigned actors to occupy either a higher or lower status position within their group and further randomly assigned each group to one of two treatment conditions, reflecting either moderate or large induced status differences (described below). All volunteers received a $\$ 25$ cash incentive for completing the study.

\section{Procedures}

A research assistant greeted each participant and escorted them to a private room in the lab. Participant rooms were all equipped with a desktop PC, a pair of headphones with microphones for communication between task partners, and a lavalier condenser microphone for collecting high quality vocal recordings. ${ }^{4}$ After providing informed consent, participants completed a two-phase study. In phase one, participants worked separately to complete either one or three contrived ability tests, which served as the basis for the status manipulation. In phase two, participants worked together to complete a collective group task. During the group task, participants communicated with their partners over a computer network using microphones and headphones. At no point did the participants meet face-to-face.

Phase one: status manipulation. Following previous research on small group status structures in open-interaction settings (e.g., Dippong, Kalkhoff, and Johnsen 2017), I manipulated status differences between partners using artificially induced status characteristics. I employed artificially created status characteristics rather than overt diffuse characteristics like age, education, race, and gender in order to maximize experimental control over the status structure within the group. By inducing status differences using novel characteristics, I was able to ensure that the status structure in each group was as identical as possible and unaffected by individual differences, biases, or variation in preexisting status beliefs. Furthermore, the use of artificial status differences precludes the possibility of group members inadvertently revealing information that contradicts the established basis of the status hierarchy. Substantial evidence supports the contention that artificially created status characteristics function the same as "naturally occurring" characteristics (see, 
e.g., Webster 1977; Dippong et al. 2017; Melamed et al. 2017). Accordingly, the use of artificial characteristics allows for a strong experimental design while remaining relevant to multiple social bases of status differentiation.

I created status structures within my experimental groups using contrived feedback regarding performance on pretests of either a single characteristic (condition 1) or three characteristics (condition 2). By manipulating the number of characteristics that differentiated group members, my goal was to induce larger status differences between group members in condition 2 than in condition 1 . The first test purportedly measured a characteristic related to participants' ability to solve problems intuitively. Although novel in its application, the test involved a slightly modified version of a well-established problem-solving task known as "Lost at Sea" (see Kalkhoff et al. [2020] for a description of Lost at Sea). The remaining two tests-called contrast sensitivity and meaning insight-have been used extensively in decades of research on small group status structures (see Moore [1968] for an overview of contrast sensitivity and Webster [1977] for an overview of meaning insight). Instructions for all three pretests emphasized that they are well-established and valid measures of socially relevant characteristics.

All three tests had similar properties, in that participants were led to believe that there was a correct solution to the problems in each test, but the problems were ambiguous enough that participants could not accurately gauge their own performance. Using such tasks, it is possible to convince participants of the validity and accuracy of practically any score, making them "an efficient means of creating and randomly assigning states of specific status characteristics" (Berger and Fisek 1970:293). Additionally, task instructions informed participants that the three ability tests were unrelated to such characteristics as race and gender. This ensures that any status differentiation between participants results entirely from experimentally manipulated characteristics. To guarantee standardization, participants received all instructions and completed the tests via computer.

After participants completed the pretest(s), the computer program provided participants with contrived feedback regarding their performance, as well as their partner's performance. To ensure that participants paid attention to the scores, they manually recorded both their scores and their partner's scores on a form we provided. Again, participants in condition 1 completed a single pretest related to one characteristic. To create status differentiation, the computer program informed group members that the individual who was randomly assigned to the lower status position scored in the "fair" range (on a scale consisting of poor, fair, average, good, and excellent) and that the higher-status actor scored in the "excellent" range. Participants in condition 2 completed the problem-solving ability test, as well as the contrast sensitivity and meaning insight tests. I assigned scores on these tests consistently across all three ability tests such that group members received feedback that the actor assigned to the higher status position scored substantially higher than the lower status actor. Higher status group members received scores on both tests that fell within the "superior" range, whereas lower status actors received scores in the "average" range. Overall, higher status actors in condition 2 were consistently advantaged across three separate characteristics. Table 1 presents the manipulated status structures of dyads in conditions one and two. 
Table 1: Dyadic status structures by condition

\begin{tabular}{lcccc}
\hline & \multicolumn{2}{c}{ Condition 1 } & \multicolumn{2}{c}{ Condition 2 } \\
\hline Manipulated Status Position & Lower Status & Higher Status & Lower Status & Higher Status \\
\hline Status Characteristics & - & + &,,--- &,,+++ \\
\hline
\end{tabular}

Status differences manipulated using either one or three artificial characteristics. Signs denote advantage or disadvantage for each status characteristic.

Phase two: group task. In the second phase, participants worked together in an open-interaction format to complete a collective decision-making task. Instructions for the group task informed participants that the task was designed to assess how the characteristics measured in phase one of the study affected the ways that they formed decisions together. Each group completed the "Lost on the Moon" task, an activity that is commonly used to examine interaction and communication in small groups (e.g., Cheng et al. 2013).

The Lost on the Moon activity involves asking group member to imagine being stranded together on the surface of the moon with a list of 15 items (e.g., oxygen tanks, nylon rope, water) salvaged from a supply ship. To complete the task, participants rank ordered the 15 items in terms of importance for survival while awaiting rescue. To emphasize that the task has a correct answer, instructions informed participants that they would receive a group score comparing their group rankings with the optimal item ranking determined by NASA. Participants first privately recorded their initial rankings of the items and used these rankings as a basis for discussion when they collaborated with their task partner.

Group members communicated through headphones over a network to complete the decision-making task. Using high quality condenser microphones, I digitally recorded conversations on a central control PC using a Roland Quad-Capture USB mixer and Audacity software, at a sampling rate of $48,000 \mathrm{~Hz}$. Prior to connecting participants to the communication network, the research assistant advised them to discuss only the assigned task and to refrain from introductions and talk unrelated to the task. A review of audio files revealed that all groups conformed to the request. During interaction, group members communicated vocally but never saw each other. Instructions asked groups to reach consensus on their rankings within 15 minutes, and all groups successfully reached consensus in the allotted time. The software recorded participants' voices on separate audio channels, and because they were isolated in separate rooms, this ensured that each track contained only a single actor's voice.

Following the group task, participants completed a posttest questionnaire that contained manipulation check items and assessed their subjective impressions of each other's status. Next, they completed a face-to-face interview in which research assistants evaluated the extent to which participants took the group task seriously and probed for any suspicion regarding experimental procedures. Lastly, participants were debriefed regarding areas of deception and received their payment. On average, experimental sessions lasted approximately one hour and 15 minutes. 


\section{Measures}

Dependent variable. To assess vocal accommodation, I employ AAR scores derived from recordings of group interactions. Following established procedures laid out by Dippong and Kalkhoff (2018), calculating AAR involves four relatively simple steps. First, I divided the audio recordings for each speaker into equal temporal segments, corresponding roughly to the beginning, middle, and end of conversation. This process produced six speech segments (three for each speaker). Second, using SpectraPlus software, I performed FFT analysis on each of the six temporal segments. Third, I included values for all six FFT segments in a single principle component factor analysis model with varimax rotation. This model reliably produces a twofactor solution. Fourth, I calculated the mean of each group member's rotated factor loadings on the first factor, which captures relative stability (Kalkhoff et al. 2017; Dippong and Kalkhoff 2018). AAR then captures the average stability of each actor's paraverbal frequencies, relative to their task partner, across the duration of the discussion. To reiterate, higher AAR values represent greater relative paraverbal stability, which I hypothesize is associated with occupying a higher status position.

Independent variables. I test the relationship between status and accommodation in two ways, first with a variable denoting each group member's position within the manipulated status structure, and second with a variable capturing participants' subjective impression of each other's status. For models examining the effects of manipulated position, I employ a dichotomous categorical variable $(0=$ lower status, 1 = higher status). A significant positive relationship between status position and AAR would provide support for my hypothesis.

For subjective impressions of status, I use a measure derived from two items embedded in the poststudy questionnaire. Participants responded to two items, presented toward the beginning and the end of the questionnaire, respectively: (1) "My partner had high status in the discussion" and (2) "I had high status in the discussion." Responses to the two items were recorded using a Likert-type scale ranging from $(1=$ strongly disagree $)$ to $(9=$ strongly agree $)$. To calculate subjective status perceptions, I created a variable in which I subtracted perceptions of task partner from perceptions of self. Positive values denote a perceived status advantage for self, and negative values represent a perceived advantage for task partner.

Control variables. My analyses also include two different sets of control variables to account for additional theoretically relevant characteristics of participants' speech, as well as demographic characteristics of the participants. For speech characteristics, I include controls for vocal pitch (produced during FFT analyses) and participants' total speaking time in seconds. In terms of demographic characteristics, I employ dichotomous variables for participant sex $(1=$ female $)$, race $(1=$ white $)$, and academic standing $(1=$ greater than freshman $){ }^{5}$ I also include a continuous measure of participant age.

Variables for testing measurement sensitivity. To examine the extent to which greater magnitudes of status differentiation produce larger differences in vocal accommodation, I estimate models that include two additional variables. First, I include a dichotomous indicator for experimental condition $(0=$ condition 1 , one status characteristic; $1=$ condition 2, three status characteristics). And second, 
Table 2: Bivariate correlations demonstrating convergence in FFT values ( $N=41$ groups)

\begin{tabular}{lccc}
\hline Segment & Condition 1 & Condition 2 & Total Sample \\
\hline Beginning & 0.562 & 0.463 & 0.507 \\
& $(0.244)$ & $(0.240)$ & $(0.245)$ \\
Middle & 0.536 & 0.471 & 0.499 \\
& $(0.218)$ & $(0.298)$ & $(0.266)$ \\
End & 0.337 & 0.437 & 0.393 \\
& $(0.343)$ & $(0.251)$ & $(0.297)$ \\
Average & 0.484 & 0.457 & 0.469 \\
& $(0.198)$ & $(0.210)$ & $(0.204)$ \\
\hline
\end{tabular}

for both the manipulated position and the subjective status models, I include a term for the interaction between status and condition. A positive and significant interaction effect would provide evidence of sensitivity to status differences of varying magnitudes.

\section{Analytic Strategy}

I utilize generalized estimating equations (GEE) to test my hypotheses. GEE produces parameter estimates based on population averaged models and is preferable to standard linear mixed models when comparing the effects of a manipulation or intervention between two or more groups (Hubbard et al. 2010). Importantly, GEE accounts for the nesting of participants within groups, is not constrained by the same distributional parameters as linear mixed modeling, and produces estimates that are generally robust against misspecification of the underlying error covariance structure (Overall and Tonidandel 2004).

\section{Results}

The primary goal of the present study is to test the causal relationship between status and vocal accommodation within the paraverbal band of the voice. In addressing these goals, I analyze data from 41 out of the original 46 dyadic groups (82 participants). My analyses exclude data from three dyads based on group members' failure to converge in their paraverbal vocal frequencies. As stated above, as a measure of accommodation, AAR assumes convergence between actors. As a first step in my analyses, I verified convergence by examining bivariate correlations between group members' vocal frequencies across all three temporal segments of the interaction. I excluded groups when they met both of the following criteria: (1) FFT values demonstrated a significant inverse bivariate relationship within at least two temporal speech segments and (2) the average Pearson correlation coefficient across all three temporal segments was negative. Table 2 presents average correlation coefficients for the three temporal segments. As seen in Table 2, all groups in the analytic sample demonstrate a clear pattern in paraverbal convergence.

I excluded from analysis an additional two dyads in which a group member expressed well-articulated suspicions regarding the status manipulation. Because 
Table 3: Descriptive statistics $(N=82)$

\begin{tabular}{lccr}
\hline & Total Sample & Lower Status & Higher Status \\
\hline Vocal Stability (AAR) & 0.590 & 0.447 & $0.733^{\dagger}$ \\
Vocal Pitch & $(0.284)$ & $(0.279)$ & $(0.208)$ \\
& 244.952 & 247.075 & 242.829 \\
Speaking Time (in seconds) & $(231.391)$ & $(218.751)$ & $(231.391)$ \\
& 203.122 & 174.073 & $232.171^{\dagger}$ \\
Subjective Status Perceptions & $(117.742)$ & $(95.403)$ & $(131.306)$ \\
& -0.098 & -1.146 & $0.951^{\dagger}$ \\
& $(1.985)$ & $(1.740)$ & $(1.642)$ \\
\hline
\end{tabular}

$+p<0.01$ (paired-samples $t$ test; denotes significant differences between higher and lower status participants).

AAR is an inherently relative measure that combines data from all group members, I exclude data from an entire group even when only one member expressed suspicion. My total exclusion rate is 10.87 percent, which is consistent with other experimental research on status in groups (see Dippong 2012). My analytical sample includes 18 groups in condition $1(n=36)$ and 23 groups in condition $2(n=46)$.

In terms of sample characteristics, participants reported an average age of 20.5 years. Approximately 43 percent of the sample identified as white, non-Hispanic. Regarding other characteristics, 41.5 percent of participants were freshmen, and 61 percent identified as female. A series of planned comparisons reveal no significant differences in age, self-reported race/ethnicity, and academic level between actors assigned to higher and lower status positions. Stated differently, random assignment distributed these characteristics evenly between comparison groups. Although the overall sample consists of a significantly greater proportion of female participants (chi-square $=4.878 ; d f=1 ; p=0.027$ ), the use of same-sex dyads precludes the possibility that sex differences between group members affected vocal accommodation patterns within groups.

Table 3 presents descriptive statistics for vocal characteristics and subjective status perceptions. Actors assigned to the higher status position produced larger AAR values (paired-sample $t=4.116 ; d f=40 ; p<0.01$ ) and spent more time speaking than their partners did (paired-sample $t=11.683 ; d f=40 ; p<0.01$ ). Contrary to what prior researchers have found, participants in my study demonstrated no significant differences in their vocal pitch. Lastly, group members' subjective perceptions reflect the intended status ordering.

\section{Manipulation Check}

To assess the effectiveness of the status manipulation, I employed three questionnaire items drawn from Zeller and Warnecke's (1973) general performance expectations index. The Zeller and Warnecke index does not directly assess status, but performance expectations represent a closely related phenomenon (Berger et al. 1977), making the measure suitable for inferring status differences. Furthermore, Driskell and Mullen (1988) provide evidence from meta-analysis that the Zeller and 
Table 4: Manipulation check: paired-samples $t$ tests comparing self-reported general performance expectations

\begin{tabular}{lccc}
\hline & Lower Status & Higher Status & Comparison \\
\hline Condition 1 & 6.815 & 7.389 & $t=3.091$ \\
& $(1.139)$ & $(0.826)$ & $d f=35$ \\
Condition 2 & & & $p<0.01$ \\
& 6.659 & 7.558 & $t=4.232$ \\
& $(1.177)$ & $(0.869)$ & $d f=45$ \\
Full Sample & & & $p<0.01$ \\
& 6.728 & 7.484 & $t=5.228$ \\
& $(1.156)$ & $(0.850)$ & $d f=81$ \\
& & & $p<0.01$ \\
\hline
\end{tabular}

Warnecke index reliably differentiates between actors of differing status. Participants rated themselves and their task partner using nine-point Likert-type scales that assessed perceptions of each actor's ability in situations in general, intelligence, and ability to do "other things that count in the world." I calculated expectation scores by taking the mean of the three items.

Table 4 presents the means, standard deviations, and paired-samples $t$ tests comparing group members' expectations for both group members. In both treatment conditions, participants reported significantly higher expectations for actors in the higher status position versus the lower status position. Additionally, the mean difference between higher and lower status actors is 56.6 percent higher in condition 2 compared with condition 1 . This suggests that the manipulation successfully produced the intended status ordering both within groups and across conditions.

\section{Analysis 1: Manipulated Status Position}

I employ GEE using STATA's xtgee command with robust standard errors. ${ }^{6}$ To select the appropriate error covariance structure for my models, I employ Cui's (2007) quasi information criteria for GEE (QIC). Although independent and exchangeable covariance structures produced nearly identical parameter estimates, QIC results favored the exchangeable structure (independent QIC = 21.789; exchangeable QIC $=18.700$ ).

Table 5 presents GEE models predicting vocal accommodation from manipulated status position ( 1 = higher status). Looking first at model 1 , we see that status significantly predicts vocal accommodation and that the relationship is in the expected direction. Model 2 introduces additional vocal factors that may influence the relationship between status and accommodation. Importantly, status maintains a significant effect when controlling for vocal pitch and speaking time. Vocal pitch is significantly and positively related to accommodation, and speaking time has no significant effect. The introduction of demographic controls in model 3 has minimal effect on the model; status and vocal pitch remain significant predictors of vocal accommodation. Overall, GEE results provide strong support for hypothesis $1 .{ }^{7}$

Regarding hypothesis 2, the last two models in Table 5 examine the effects of study condition and the binary interaction term between status position and 
Table 5: GEE predicting vocal accommodation from manipulated status position $(N=82)$

\begin{tabular}{|c|c|c|c|c|c|}
\hline & Model 1 & Model 2 & Model 3 & Model 4 & Model 5 \\
\hline Constant & $\begin{array}{r}0.447^{\dagger} \\
(0.044)\end{array}$ & $\begin{array}{c}0.448^{+} \\
(0.043)\end{array}$ & $\begin{array}{c}0.475^{\dagger} \\
(0.060)\end{array}$ & $\begin{array}{r}0.540^{\dagger} \\
(0.056)\end{array}$ & $\begin{array}{c}0.611^{\dagger} \\
(0.063)\end{array}$ \\
\hline Status & $\begin{array}{r}0.286^{+} \\
(0.069)\end{array}$ & $\begin{array}{r}0.283^{\dagger} \\
(0.068)\end{array}$ & $\begin{array}{r}0.291^{\dagger} \\
(0.069)\end{array}$ & $\begin{array}{r}0.297^{+} \\
(0.069)\end{array}$ & $\begin{array}{c}0.151 \\
(0.100)\end{array}$ \\
\hline Vocal Pitch & & $\begin{array}{r}0.029^{+} \\
(0.009)\end{array}$ & $\begin{array}{r}0.028^{+} \\
(0.009)\end{array}$ & $\begin{array}{r}0.030^{\dagger} \\
(0.009)\end{array}$ & $\begin{array}{r}0.030^{+} \\
(0.009)\end{array}$ \\
\hline Speaking Time & & $\begin{array}{c}0.007 \\
(0.017)\end{array}$ & $\begin{array}{c}0.000 \\
(0.017)\end{array}$ & $\begin{array}{c}-0.008 \\
(0.015)\end{array}$ & $\begin{array}{r}-0.006 \\
(0.015)\end{array}$ \\
\hline Sex & & & $\begin{array}{r}-0.032 \\
(0.030)\end{array}$ & $\begin{array}{c}-0.046 \\
(0.029)\end{array}$ & $\begin{array}{c}-0.044 \\
(0.029)\end{array}$ \\
\hline Age & & & $\begin{array}{c}0.005 \\
(0.008)\end{array}$ & $\begin{array}{c}0.005 \\
(0.008)\end{array}$ & $\begin{array}{c}0.004 \\
(0.008)\end{array}$ \\
\hline Race (1 = White) & & & $\begin{array}{c}-0.011 \\
(0.040)\end{array}$ & $\begin{array}{c}-0.021 \\
(0.033)\end{array}$ & $\begin{array}{c}-0.018 \\
(0.033)\end{array}$ \\
\hline Academic Standing & & & $\begin{array}{r}-0.010 \\
(0.055)\end{array}$ & $\begin{array}{r}-0.016 \\
(0.047)\end{array}$ & $\begin{array}{r}-0.018 \\
(0.046)\end{array}$ \\
\hline Condition & & & & $\begin{array}{r}-0.091^{\dagger} \\
(0.030)\end{array}$ & $\begin{array}{r}-0.219^{+} \\
(0.078)\end{array}$ \\
\hline Status $\times$ Condition & & & & & $\begin{array}{r}0.255^{*} \\
(0.130)\end{array}$ \\
\hline
\end{tabular}

$+p<0.01 ; * p<0.05$.

Numbers in parentheses are robust standard errors.

condition. As expected, status position maintains a significant effect on vocal accommodation when controlling for experimental condition. More importantly, model 5 shows a significant interaction between status and condition. Looking at the status by condition interaction term, the coefficient is positive and significant. In other words, the relationship between status and accommodation is conditioned by the magnitude of the status difference. Larger status differences produce larger differences in AAR, providing support for hypothesis 2.

To further illustrate the effects of status and condition on vocal accommodation, Figure 1 presents the simple slopes plot of the interaction. From the slopes plot, it appears that larger status differences lead to greater differences in vocal accommodation primarily by producing lower AAR scores in lower status group members while having virtually no effect on the AAR scores of higher status actors. Indeed, lower status actors in condition 2 produced significantly lower AAR scores than those in condition 1 (independent samples $t=2.478 ; p=0.017$, two-tailed). For actors in high status positions, average AAR did not vary by condition (independent samples $t=-.761 ; p=0.451$, two-tailed). This result is consistent with what we might expect, given that that higher status actors demonstrate relatively little variability in the paraverbal band in the first place. In other words, higher status actors demonstrated comparable levels of stability, regardless of the status distance between them and their task partners. Conversely, lower status actors 


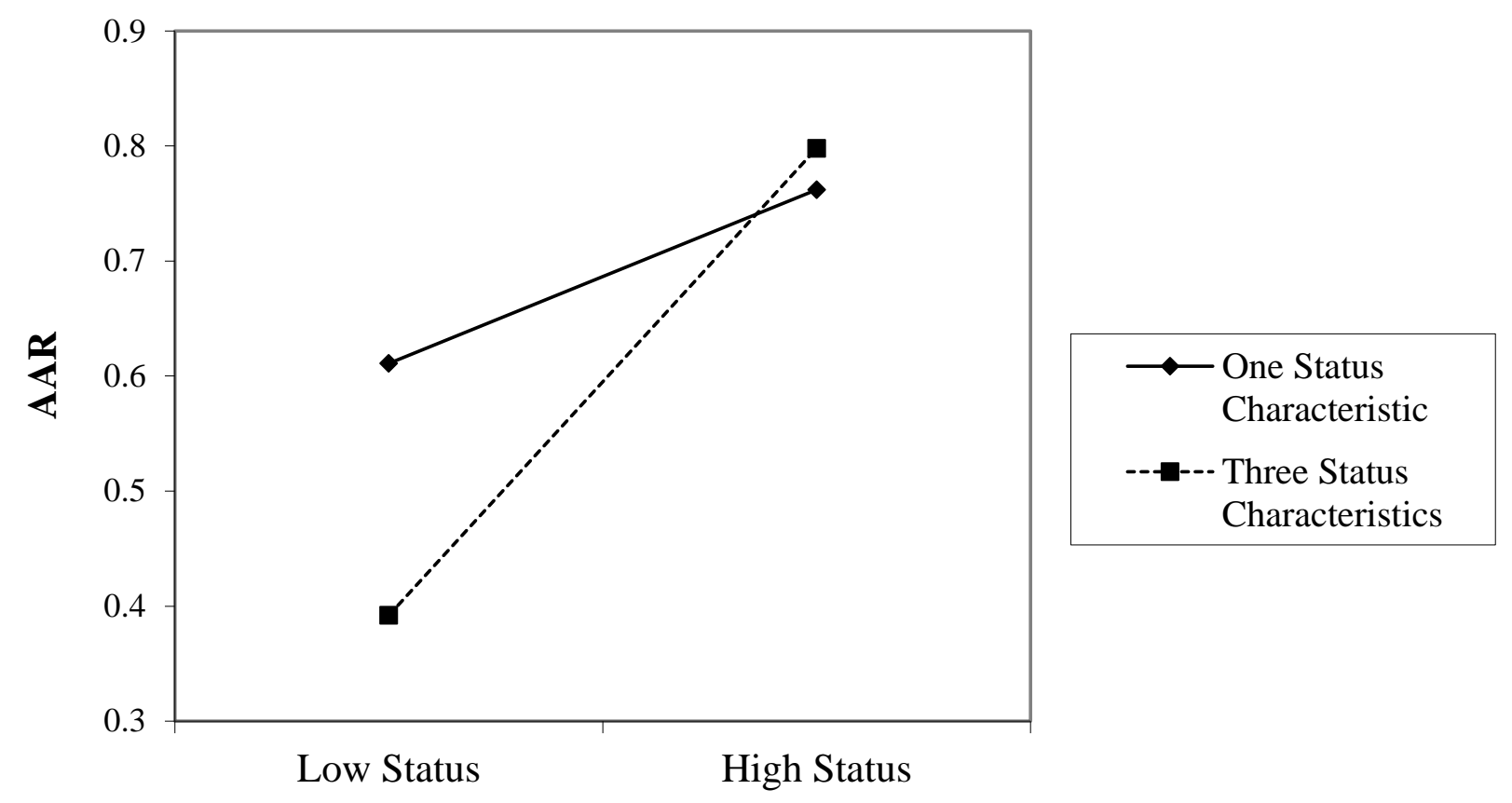

Figure 1: Simple slopes plot with binary interaction between relative status position and magnitude of manipulated status differences.

revealed substantially greater variability (and hence lower AAR) as their relative status decreased.

\section{Analysis 2: Subjective Status Perceptions}

Having demonstrated a causal relationship between relative status and vocal accommodation using the objective status structure of an interaction, I turn next to the relationship between vocal accommodation and group members' subjective perceptions of each other's relative status. As can be seen in Table 6, the pattern of results is nearly identical to those obtained using group members' manipulated status position. The relationship between status perceptions and vocal accommodation is positive and significant in model 1 , and the relationship holds up after accounting for additional vocal characteristics and demographic factors in models 2 and 3. These results lend further support to hypothesis 1; whether modeled in terms of an objective indicator of group status structure or in terms of group members' self-reported status perceptions, higher status actors produce significantly higher AAR values.

There is one very noteworthy difference between the effects of manipulated and subjective status differences, namely, that the interaction between subjective status perceptions study condition is not significant. This finding does not, however, necessarily undermine the results obtained based on manipulated status position. Unlike the dichotomous indicator of status that I used to model objective manipulated 
Table 6: GEE predicting vocal accommodation from subjective status perceptions ( $N=82)$

\begin{tabular}{|c|c|c|c|c|c|}
\hline & Model 1 & Model 2 & Model 3 & Model 4 & Model 5 \\
\hline Constant & $\begin{array}{r}0.593^{\dagger} \\
(0.017)\end{array}$ & $\begin{array}{c}0.593^{+} \\
(0.015)\end{array}$ & $\begin{array}{c}0.615^{\dagger} \\
(0.044)\end{array}$ & $\begin{array}{c}0.673^{+} \\
(0.038)\end{array}$ & $\begin{array}{r}0.672^{+} \\
(0.039)\end{array}$ \\
\hline Status Perceptions & $\begin{array}{c}0.031^{\dagger} \\
(0.011)\end{array}$ & $\begin{array}{r}0.037^{\dagger} \\
(0.011)\end{array}$ & $\begin{array}{r}0.038^{\dagger} \\
(0.011)\end{array}$ & $\begin{array}{r}0.032^{+} \\
(0.011)\end{array}$ & $\begin{array}{c}0.031^{*} \\
(0.015)\end{array}$ \\
\hline Vocal Pitch & & $\begin{array}{c}0.033^{+} \\
(0.008)\end{array}$ & $\begin{array}{r}0.029^{\dagger} \\
(0.008)\end{array}$ & $\begin{array}{r}0.030^{+} \\
(0.008)\end{array}$ & $\begin{array}{r}0.030^{+} \\
(0.008)\end{array}$ \\
\hline Speaking Time & & $\begin{array}{c}0.004 \\
(0.017)\end{array}$ & $\begin{array}{r}-0.004 \\
(0.017)\end{array}$ & $\begin{array}{r}-0.010 \\
(0.014)\end{array}$ & $\begin{array}{r}-0.010 \\
(0.014)\end{array}$ \\
\hline Sex & & & $\begin{array}{r}-0.038 \\
(0.028)\end{array}$ & $\begin{array}{r}-0.050 \\
(0.028)\end{array}$ & $\begin{array}{c}-0.049 \\
(0.028)\end{array}$ \\
\hline Age & & & $\begin{array}{r}-0.000 \\
(0.009)\end{array}$ & $\begin{array}{c}0.001 \\
(0.009)\end{array}$ & $\begin{array}{c}0.001 \\
(0.009)\end{array}$ \\
\hline Race (1 = White) & & & $\begin{array}{c}-0.028 \\
(0.040)\end{array}$ & $\begin{array}{c}-0.033 \\
(0.033)\end{array}$ & $\begin{array}{c}-0.033 \\
(0.033)\end{array}$ \\
\hline Academic Standing & & & $\begin{array}{c}0.024 \\
(0.050)\end{array}$ & $\begin{array}{c}-0.014 \\
(0.043)\end{array}$ & $\begin{array}{c}0.015 \\
(0.046)\end{array}$ \\
\hline Condition & & & & $\begin{array}{r}-0.077^{\dagger} \\
(0.028)\end{array}$ & $\begin{array}{r}-0.077^{\dagger} \\
(0.027)\end{array}$ \\
\hline Interaction & & & & & $\begin{array}{c}0.002 \\
(0.021)\end{array}$ \\
\hline
\end{tabular}

$+p<0.01 ; * p<0.05$.

Numbers in parentheses are robust standard errors.

status position, the subjective indicator already accounts for different magnitudes of status differentiation between conditions. In condition 1 (one status characteristic), the mean difference between higher and lower status actors' subjective perceptions was $1.56(t=-2.881 ; p<0.01)$. Conversely, for condition 2 (three status characteristics) the mean difference in status perceptions was $2.52(t=-4.964 ; p<0.01)$. Thus, the measure of status itself is already sensitive to magnitudes of differentiation, and the interaction term is essentially redundant.

\section{Summary}

Taken as a whole, analysis of objective indicators of status position (as shown in Table 5) and subjective self-reports of status perceptions (as shown in Table 6) both provide clear evidence that differences in vocal accommodation between interactants are driven by differences in status. Most importantly, however, my analyses show that as a measure of vocal accommodation, AAR captures the internal status dynamics of a group. Whereas previous studies have relied on the perceptions of external observers, my study shows that AAR reflects the definition of the situation as determined by the interactants themselves. My results also show that vocal accommodation is a useful measure of status in groups where the status structure is known and when the status structure is modeled using post hoc subjective measures of status. Furthermore, I have provided initial evidence that AAR can be useful for 
assessing the magnitude of status differences between actors, with smaller status differences reflected in smaller differences in AAR values. In the next section, I summarize the primary contributions of the present study, discuss the potential for vocal accommodation as an unobtrusive measure of status, and outline useful future directions for research on vocal accommodation and status.

\section{Discussion}

In the present study, I have provided an experimental test of the causal relationship between status and vocal accommodation. The research design I employed represents a substantial step forward in our understanding of vocal accommodation processes in two ways. First, whereas prior research on vocal accommodation has utilized post hoc self-report data to infer status differences at the end of an interaction, the present study allows for strong causal inference by manipulating status differences prior to interaction and assessing how such differences shape vocal dynamics. Second, prior studies on vocal accommodation have employed panels of external raters to infer the status structure of a group they observe. The present study uses vocal accommodation to model status differences using only the manipulated structure of the group, the perceptions of interacting actors, and audio recordings of group interactions. In this way, the results provide new insight on how vocal accommodation reflects the internal structure and dynamics of status-differentiated small groups.

Aside from providing experimental evidence to support decades of theoretical and empirical work on vocal accommodation, my results also help address some open questions regarding the social bases of vocal accommodation. Specifically, whereas some studies have found evidence connecting vocal accommodation to hierarchies of status (e.g., Gregory and Webster 1996; Gallagher et al. 2005), Kalkhoff et al. (2017) found that in a set of televised interviews, vocal accommodation predicted observers' perceptions of dominance, but not prestige. Regarding these results, Kalkhoff and colleagues (2017:351) note that "the interviews were to some degree conflictual and in one case involved an especially heated exchange ... It is important to know whether our findings would replicate when panelists watch or listen to interactions that are more cooperative in nature." By examining vocal accommodation in groups that were both cooperative and explicitly organized according to status differences, we can be confident that vocal accommodation does indeed reflect status differences.

This argument is bolstered by the fact that I found no significant differences between group members in terms of their dominance perceptions of each other (paired-sample $t=-1.134, d f=81, p=0.26$ ). Given that participants in the present study demonstrate significant differences in status perceptions but no significant differences in dominance perceptions, it seems quite unlikely that the patterns of vocal accommodation I observed can be attributed to structures of dominance and not status. It is important to note that whereas I analyze how status affects group members' status perceptions, Kalkhoff and colleagues focus on the perceptions of observers. Accordingly, the analyses are not necessarily contradictory. 
Although my results are based on interaction in a controlled laboratory setting, the phenomenon is not restricted to such conditions. The decision to employ a laboratory experiment was based solely on obtaining the high degree of internal validity necessary to test causal relationships. In terms of broader generalizability, the results of any social experiment are applicable to naturalistic settings to the extent that both the experiment and the social situation meet the same theoretical conditions (Zelditch 1969; Webster and Kervin 1971). In other words, the use of a laboratory setting for this study does not limit the applicability of its findings and methods of analysis to only laboratory settings. Existing studies examining vocal accommodation using secondary data from debates (Gregory and Gallagher 2002; Kalkhoff and Gregory 2008) and interviews (Gallagher et al. 2005; Kalkhoff et al. 2017) attest to the usefulness of the approach for examining everyday interactions. The internal validity of laboratory experimentation only serves to increase confidence that the relationship between vocal accommodation and status really operates as the theoretical model has proposed.

\section{Vocal Accommodation as an Unobtrusive Measure of Status}

Sociologists interested in studying inequalities related to group-level status structures have at their disposal a variety of experimental, observational, and surveybased tools for assessing behavioral outcomes related to interaction in stratified social settings. Given its demonstrated relationship to status, researchers can employ vocal accommodation as a stand-alone measure of status (e.g., the present study) or as a supplementary measure that can be used alongside other existing measures (e.g., to analyze recordings collected for other purposes). Furthermore, vocal accommodation is useful both as a dependent variable produced by status processes and an independent variable predicting other behaviors.

As a measure of vocal accommodation, AAR possess two broad properties that make it a particularly valuable research tool for status researchers. First, AAR captures processes that occur outside of conscious awareness. Despite the fact that behavioral accommodation is a fundamental aspect of interaction, people are rarely aware that it is occurring (Condon and Sander 1974). This is even more so the case with vocal accommodation, in that it occurs within a range of vocal frequencies that interactants do not consciously attend to and that speakers do not consciously manipulate (Gregory et al. 2009). As such, measures of vocal accommodation bypass individual self-presentation motives. Research participants can manipulate responses on a questionnaire, for example, to ensure presenting a socially desirable self; conversely, existing evidence suggests that such manipulation is not possible (or at least extremely difficult) with vocal accommodation.

Second, researchers can collect vocal data relatively easily and unobtrusively. Given the ubiquity of audio and video recording in research settings, many researchers already collect the necessary data to conduct the kind of analysis I presented. Simply providing members of discussion groups with microphones opens an impressive array of analytical options. Technological and methodological advancements make it increasingly easy to collect vocal data, including methodological tools developed specifically for sociological research (e.g., Parker et al. 2018). 
And despite the fact that recording people can activate self-presentation motives, participants are unlikely to guess the purpose of the recording, and even then, it is unlikely that they would be able to significantly alter their paraverbal vocal behaviors. Furthermore, the approach is useful for analyzing any secondary data with sufficient audio quality, including vocal data from television, radio, podcasts, and websites.

It is also worth noting that generating quantitative measures of vocal accommodation requires relatively simple methods and analytical procedures. As stated above, step-by-step procedures for collecting and analyzing original and secondary data are available in multiple places (see especially Dippong and Kalkhoff 2018; Kalkhoff et al. 2017). Likewise, the hardware and software necessary to conduct vocal spectral analysis is relatively inexpensive, and open source software has made many of the basic necessities easily and freely accessible.

\section{Future Directions}

The present study represents a potentially substantial step forward in the sociological understanding of how vocal processes reflect social structure, although two noteworthy questions remain. To this end, the vast majority of research on vocal accommodation has focused on interacting dyads. More work is needed to assess how the process operates in larger interaction groups, as well as the extent to which quantitative indicators require modification for larger and more complex settings. Additionally, if vocal accommodation is to be widely useful, it would be beneficial to examine how vocal accommodation relates to other status-related behaviors, such as influence patterns in experimental task groups. Future research on vocal accommodation should involve collecting multiple behavioral indicators to allow for comparisons of the general properties of the measures.

\section{Conclusion}

In this article, using experimental methods, I have demonstrated the causal relationship between vocal accommodation and small group status structures. Furthermore, I have argued that vocal accommodation is useful as an unobtrusive measure of status. Although on the surface, it may seem that vocal accommodation is a phenomenon best suited for use in social psychological research, its applicability is in fact, much broader. For scholars who study organizations, vocal accommodation is useful for understanding how work groups embedded in larger organizations make decisions together. For those interested in biosocial processes, vocal accommodation offers a mechanism to examine the interplay between social structure and bodily processes. And for scholars who study social dynamics related to race or gender, vocal accommodation provides a way to assess inequalities using measures that bypass conscious motivations to act in socially desirable ways. As a means to measure and model status-based behaviors, vocal accommodation serves as a potentially valuable tool for advancing the scientific understanding how social structure impinges on interaction. 


\section{Notes}

1 Scholars have variously referred to this framework as accommodation theory (Giles and Smith 1979), speech accommodation theory (Giles et al. 1987), and communication accommodation theory (Giles and Coupland 1991). I employ the term accommodation theory to highlight a broad focus on behavioral convergence.

2 Accommodation theory acknowledges two potential forms of behavioral adaptation: convergence occurs when actors behave to minimize differences, and divergence occurs when actors seek to emphasize individual distinctiveness (Gallois et al. 2005). The vocal accommodation model presented here applies solely to interactions in which convergence occurs. That is, the logic of current measures of accommodation assume vocal convergence (Dippong and Kalkhoff 2018).

3 For readers interested in the technical details of vocal analysis, Dippong and Kalkhoff (2018) offer a thorough overview of the most common processes for collecting audio data, for preparing data for analysis, and for assessing vocal outcomes. Similarly, Kalkhoff and colleagues (2017, online supplement) provide step-by-step instructions, as well as specific software settings used for calculating the most common measures of vocal accommodation.

4 The present study utilized Sennheiser PC 151 microphone headsets and Audio-Technica AT831B cardioid lavalier condenser microphones.

5 I estimated additional models in which I include more fine-grained racial and academic categories, and the analyses produced the same substantive results as those reported here.

6 I replicated these analyses employing a mixed model approach; using the xtmixed command in STATA with random effects for group membership, mixed-effects models produced substantively identical results to the GEE models reported here. Analyses available from author.

7 Data and codebook to replicate all analyses presented here are available at https://doi . org/10.7910/DVN/CPK5QI.

\section{References}

Bachorowski, Jo-Anne. 1999. “Vocal Expression and Perception of Emotion." Current Directions in Psychological Science 8(2):53-57. https : //doi .org/10.1111/1467-8721.00013

Baus, Cristina, Phil McAleer, Katherine Marcoux, Pascal Belin, and Albert Costa. 2019. "Forming Social Impressions from Voices in Native and Foreign Languages." Scientific Reports 9(1):1-14. https://doi .org/10.1038/s41598-018-36518-6

Berger, Joseph, and M. Hamit Fisek. 1970. "Consistent and Inconsistent Status Characteristics and the Determination of Power and Prestige Orders." Sociometry 33:287-304. https : //doi.org/10.2307/2786159

Berger, Joseph, M. Hamit Fisek, Robert Z. Norman, and Morris Zelditch Jr. 1977. Status Characteristics and Social Interaction. New York: Elsevier.

Cheng, Joey T., Jessica L. Tracy, Tom Foulsham, Alan Kingstone, and Joseph Henrich. 2013. "Two Way to the Top: Evidence the Power and Prestige are Distinct Yet Viable Avenues to Social Rank and Influence." Journal of Personality and Social Psychology 104(1):103-25. https://doi.org/10.1037/a0030398 
Cheng, Joey T., and Jessica L. Tracy. 2014. "Toward a Unified Science of Hierarchy: Dominance and Prestige Are Two Fundamental Pathways to Human Social Rank." Pp. 3-27 in The Psychology of Social Status, edited by Joey T. Cheng, Jessica L. Tracy, and Cameron Anderson. New York: Springer. https ://doi .org/10.1007/978-1-4939-0867-7_1

Condon, William S. 1986. "Communication, Rhythm, and Structure." Pp. 55-77 in Rhythm in Psychological, Linguistic, and Musical Processes, edited by J. Evans and M. Clynes. Springfield, IL: Thomas.

Condon, William S., and William D. Ogston. 1967. "A Segmentation of Behavior." Journal of Psychiatric Research 5(3):221-35. https : //doi .org/10.1016/0022-3956 (67) 90004-0

Condon, William S., and Louis W. Sander. 1974. "Neonate Movement is Synchronized with Adult Speech: Interactional Participation and Language Acquisition." Science 183(4120):99-101. https: //doi.org/10.1126/science.183.4120.99

Cui, James. 2007. "QIC Program and Model Selection in GEE Analysis." Stata Journal 7(2):209-20. https://doi.org/10.1177/1536867X0700700205

Dippong, Joseph. 2012. “The Effects of Scope Condition-Based Participant Exclusion on Experimental Outcomes in Expectation States Research: A Meta-Analysis." Social Science Research 41(2):359-71. https://doi .org/10.1016/j.ssresearch.2011.11.005

Dippong, Joseph, and Will Kalkhoff. 2018. "Modeling Small Group Status and Power Dynamics Using Vocal Accommodation." Advances in Group Processes 35:51-74. https : //doi.org/10.1108/S0882-614520180000035003

Dippong, Joseph, Will Kalkhoff, and Eugene Johnsen. 2017. "Status, Networks, and Opinions: An Experimental Investigation." Social Psychology Quarterly 80(2):153-173. https ://doi . org/10.1177/0190272517693211

Driskell, James E., and Brian Mullen. 1988. "Expectations and Actions." Pp. 399-412 in Status Generalization: New Theory and Research, edited by Murray Webster and Martha Foschi. Stanford, CA: Stanford University Press.

Gallagher, Timothy J., Stanford W. Gregory Jr., Alison J. Bianchi, Paul J. Hartung, and Sarah Harkness. 2005. "Examining Medical Interview Asymmetry using the Expectation States Approach." Social Psychology Quarterly 68(3):187-203. https ://doi .org/10.1177/ 019027250506800301

Gallois, Cindy, Tania T. Ogay, and Howard Giles. 2005. "Communication Accommodation Theory: A Look Back and a Look Ahead." Pp. 121-48 in Theorizing About Intercultural Communication, edited by W. B. Gundykunst. Thousand Oaks, CA: Sage.

Giles, Howard, and Nikolas Coupland. 1991. Language: Contexts and Consequences. Pacific Grove, CA: Brooks.

Giles, Howard, and Philip Smith. 1979. "Accommodation Theory: Optimal Levels of Convergence." Pp. 45-65 in Language and Social Psychology, edited by H. Giles and R. N. St. Clair. Baltimore, MD: University Park Press.

Gregory, Stanford W., Jr. 1983. "A Quantitative Analysis of Temporal Symmetry in Microsocial Relations." American Sociological Review 48:129-35. https : //doi .org/10. 2307/ 2095151

Gregory, Stanford W., Jr. 1986. "Social Psychological Implications of Voice Frequency Correlations: Analyzing Conversation Partner Adaptation by Computer." Social Psychology Quarterly 49(3):237-46. https://doi .org/10.2307/2786806

Gregory, Stanford W., Jr. 1994. "Sounds of Power and Deference: Acoustic Analysis of Macro Social Constraints on Micro Interaction." Sociological Perspectives 37(4):497-526. https://doi.org/10.2307/1389277 
Gregory, Stanford W., Jr., and Brian R. Hoyt. 1982. “Conversation Partner Mutual Adaptation as Demonstrated by Fourier Series Analysis." Journal of Psycholinguistic Research 11(1): 35-46. https://doi.org/10.1007/BF01067500

Gregory, Stanford W., Jr., and Stephen Webster. 1996. “A Nonverbal Signal in Voices of Interview Partners Effectively Predicts Communication Accommodation and Social Status Perceptions." Journal of Personality and Social Psychology 70(6):1231-1240. https : //doi.org/10.1037/0022-3514.70.6.1231

Gregory, Stanford W., Jr., and Timothy J. Gallagher. 2002. "Spectral Analysis of Candidates' Nonverbal Vocal Communication: Predicting U.S. Presidential Election Outcomes." Social Psychology Quarterly 65(3):298-308. https : //doi .org/10.2307/3090125

Gregory, Stanford W., Jr., and Will Kalkhoff. 2007. "Analyzing Sequences of Interactive Voice Data using Fast Fourier Transform Analysis." Mathematical Sociologist 11(1):7-11.

Gregory, Stanford W., Jr., Kelly Dagan, and Stephen Webster. 1997. “Evaluating the Relation of Vocal Accommodation in Conversation Partners' Fundamental Frequencies to Perceptions of Communication Quality." Journal of Nonverbal Behavior 21(1):23-43. https://doi.org/10.1023/A:1024995717773

Gregory, Stanford W., Jr., Stephen Webster, and Gang Huang. 1993. “Voice Pitch and Amplitude Convergence as a Metric of Quality in Dyadic Interviews." Language $\mathcal{E}$ Communication 13(3):195-217. https ://doi .org/10.1016/0271-5309(93)90026- J

Gregory, Stanford W. Jr., Brian E. Green, Robert M. Carrothers, Kelly A. Dagan, and Stephen W. Webster. 2001. "Verifying the Primacy of Voice Fundamental Frequency in Social Status Accommodation." Language \& Communication 21(1):37-60. https : //doi . org/10 . 1016/S0271-5309(00)00011-2

Gregory, Stanford W., Jr., Will Kalkhoff, Sarah K. Harkness, and Jessica L. Paull. 2009. "Targeted High and Low Speech Frequency Bands to Right and Left Ears Respectively Improve Task Performance and Perceived Sociability in Dyadic Conversations." Laterality 14(4):423-440. https : //doi .org/10.1080/13576500802598181

Hall, Edward T. 1981. Beyond Culture. New York: Doubleday.

Hubbard, Alan E., Jennifer Ahern, Nancy L. Fleischer, Mark Van der Laan, Sheri A. Lippman, Nicholas Jewell, Tim Bruckner, and William Santariano. 2010. “To GEE or Not to GEE: Comparing Population Average and Mixed Models for Estimating Associations between Neighborhood Risk Factors and Health." Epidemiology 21(4):467-74. https: //doi.org/10.1097/EDE.0b013e3181caeb90

Kalkhoff, Will, and Stanford W. Gregory Jr. 2008. "Beyond the Issues: Nonverbal Vocal Communication, Power Rituals, and 'Rope-a-Dopes' in the 2008 Presidential Debates." Current Research in Social Psychology 14(3):39-51.

Kalkhoff, Will, Joseph Dippong, and Stanford W. Gregory Jr. 2011. “The Biosociology of Solidarity." Sociology Compass 5(10):936-348. https ://doi .org/10.1111/j .1751-9020. $2011.00412 . \mathrm{x}$

Kalkhoff, Will, Shane R. Thye, and Stanford W. Gregory Jr. 2017. “Nonverbal Adaptation and Audience Perceptions of Dominance and Prestige." Social Psychology Quarterly 80(4):342-54. https://doi.org/10.1177/0190272517738215

Kalkhoff, Will, Shane R. Thye, Edward J. Lawler, Joshua Pollock, Brennan Miller, Matthew Pfeiffer, and Dudley Girard. 2015. "Affect, Behavior, and Brain Coordination in Social Exchange." Paper presented at the Annual Meeting of the American Sociological Association, August 21, Chicago, IL. 
Kalkhoff, Will, Joseph Dippong, Adam Gibson, and Stanford W. Gregory Jr. 2020. “Society in Peril? How Distance Media Communication Could be Undermining Symbolic Interaction." Pp. 317-38 in Identity and Symbolic Interaction: Deepening Foundation; Building Bridges, edited by Richard T. Serpe, Robin Stryker, and Brian Powell. New York: Springer. https://doi.org/10.1007/978-3-030-41231-9_12

Melamed, David, Will Kalkhoff, Siqi Han, and Xiangrui Li. 2017. “The Neural Bases of Status-Based Influence." Socius 3:1-10. https://doi .org/10.1177/2378023117709695

Moore, Christopher D., Joseph Dippong, and Michael S. Rejtig. 2019. "Leadership, Gender, and Vocal Dynamics in Small Groups." Current Research in Social Psychology 27:21-32.

Moore, James C., Jr. 1968. "Status and Influence in Small Group Interactions." Sociometry 1:47-63. https://doi.org/10.2307/2786479

Natale, Michael. 1975. "Social Desirability as Related to Convergence of Temporal Speech Patterns." Perceptual and Motor Skills 40(3):827-830. https://doi.org/10.2466/pms . 1975.40 .3 .827

Overall, John E., and Scott Tonidandel. 2004. "Robustness of Generalized Estimating Equation (GEE) Tests of Significance against Misspecification of the Error Structure Model." Biometrical Journal 46(2):203-13. https : //doi .org/10.1002/bimj . 200210017

Parker, John N., Edgar Cardenas, Alexander N. Dorr, and Edward J. Hackett. 2018. “Using Sociometers to Advance Small Group Research." Sociological Methods and Research, first published on May 2, 2018, as doi:10.1177/0049124118769091 https : //doi .org/10.1177/ 0049124118769091

Pitts, Mary Jane, and Cindy Galois. 2019. "Social Markers in Language and Speech." Oxford Research Encyclopedia of Psychology. https://doi.org/10.1093/acrefore/ 9780190236557.013 .300

Ruch, Hanna, Yvonne Zürcher, and Judith M. Burkart. 2018. "The Function and Mechanism of Vocal Accommodation in Humans and Other Primates." Biological Reviews 93(2):9961013. https://doi.org/10.1111/brv. 12382

Webster, Murray. 1977. "Equating Characteristics and Social Interaction: Two Experiments." Sociometry 40(1):41-50. https : //doi .org/10.2307/3033544

Webster, Murray, and John B. Kervin. 1971. "Artificiality in Experimental Sociology.” Canadian Review of Sociology and Anthropology 8(4):263-272. https://doi.org/10.1111/j . 1755-618X.1971.tb02366.x

Zelditch, Morris, Jr. 1969. "Can You Really Study an Army in the Laboratory?" Pp. 528-39 in Complex Organizations, edited by Amitai Etzioni. New York: Holt, Rinehart, and Winston.

Zeller, Richard A., and Richard B. Warnecke. 1973. "The Utility of Intervening Constructs in Experiments." Sociological Methods \& Research 2:85-110. https://doi .org/10.1177/ 004912417300200105

Acknowledgments: The research reported here was funded in whole under award W911NF17-1-0008 from the U.S. Army Research Office/Army Research Laboratory. The views expressed are those of the author and should not be attributed to the Army Research Office/Army Research Laboratory.

Joseph Dippong: Department of Sociology, University of North Carolina at Charlotte. E-mail: jdippong@uncc.edu. 